\title{
PERIODIC SOLUTIONS OF SOME LIÉNARD EQUATIONS WITH SINGULARITIES
}

\author{
PATRICK HABETS AND LUIS SANCHEZ
}

(Communicated by Kenneth R. Meyer)

ABstract. We consider the forced Liénard equation

$$
u^{\prime \prime}+f(u) u^{\prime}+g(t, u)=h(t)
$$

together with the boundary conditions

$$
u(0)=u(T), \quad u^{\prime}(0)=u^{\prime}(T),
$$

where $g$ is continuous on $\mathbf{R} \times(0,+\infty)$ and becomes infinite at $u=0$. We consider classical solutions as well as generalized solutions that can go into the singularity $u=0$. The method of approach uses upper and lower solutions and degree theory.

\section{INTRODUCTION}

Let $h \in L^{1}(0, T), f \in C(\mathbf{R}, \mathbf{R})$, and $g: \mathbf{R} \times(0,+\infty) \rightarrow \mathbf{R}$ be a Carathéodory function, $T$-periodic in the first variable. We are interested in the existence of solutions to

$$
\begin{aligned}
u^{\prime \prime}+f(u) u^{\prime}+g(t, u) & =h(t), \\
u(0)=u(T), \quad u^{\prime}(0) & =u^{\prime}(T) .
\end{aligned}
$$

Throughout we assume that:

(A) Given real numbers $0<a<b$, there exists a function $k \in L^{1}(0, T)$ such that, if $t \in[0, T]$ and $a \leq u \leq b$, we have

$$
|g(t, u)| \leq k(t) \text {. }
$$

Moreover, our assumptions are such that $u=0$ is a singularity of the "force" $g$, which becomes unbounded as $u \rightarrow 0$.

The corresponding problem in the absence of dissipation has been studied by Lazer and Solimini [4]. We use some of the ideas of that paper to obtain existence results for $(0)$. The paper is organized as follows: In $\S 2$ we state and prove a theorem on upper and lower solutions for the $W^{2,1}(0, T)$ setting; such

Received by the editors May 8, 1989.

1980 Mathematics Subject Classification (1985 Revision). Primary 34C15, 34C25.

Key words and phrases. Upper and lower solutions, singular nonlinearity.

The second author's research was supported by JNICT (contract no. 87 589). 
a result is a particular case of results obtained by Adje [1] in his doctoral dissertation. $\S 3$ deals with attractive forces; Theorem 1 provides classical solutions and Theorem 2 generalized solutions which allow collision with the singularity $u=0$; the latter are related to a concept introduced by Bahri and Rabinowitz [2]. In $\S 4$ we study repulsive forces using degree theory. This approach is very similar to Habets and Sanchez [3] where a vector case is studied using a "strong force" condition. In the scalar case such a "strong force" assumption turns out to be unnecessary.

Throughout the paper we use \|\|$_{p}$ to denote the usual norm in $L^{p}(0, T)$ $(1 \leq p \leq \infty)$.

\section{AN AUXILIARY RESULT INVOLVING LOWER AND UPPER SOLUTIONS}

Let $\alpha, \beta$ be two continuous functions in $[0, T]$ such that $\alpha(t) \leq \beta(t)$ for all $t \in[0, T]$ and set

$$
E=\{(t, x, y): 0 \leq t \leq T, \alpha(t) \leq x \leq \beta(t), y \in \mathbf{R}\} .
$$

Let $f: E \rightarrow \mathbf{R}$ be a Caratheodory function, i.e. a function with the following properties: (i) for each $x \in \mathbf{R}$ and $y \in \mathbf{R}$ the function $f(\cdot, x, y)$, with domain $\{t \in[0, T]:(t, x, y) \in E\}$, is measurable; (ii) the functions $f(\cdot, \alpha(\cdot), y)$ and $f(\cdot, \beta(\cdot), y)$ are measurable for each $y \in \mathbf{R}$; (iii) for almost every $t \in[0, T]$, the function $f(t, \cdot, \cdot)$, with domain $\{(x, y): \alpha(t) \leq x \leq \beta(t)$ and $y \in \mathbf{R}\}$ is continuous; (iv) there exists $h \in L^{1}(0, T)$ such that, for all $(t, x)$ such that $(t, x, 0) \in E$,

$$
|f(t, x, 0)| \leq h(t) .
$$

Proposition 1. Let $E$ and $f$ be as above and assume in addition that there exists $L \in L^{1}(0, T)$ such that for any $\left(t, x, y_{1}\right) \in E$ and $\left(t, x, y_{2}\right) \in E$ we have

$$
\left|f\left(t, x, y_{1}\right)-f\left(t, x, y_{2}\right)\right| \leq L(t)\left|y_{1}-y_{2}\right| \text {. }
$$

Suppose moreover that $\alpha, \beta \in W^{2,1}(0, T)$ are such that:

$$
\begin{aligned}
\alpha^{\prime \prime}(t) & \geq f\left(t, \alpha(t), \alpha^{\prime}(t)\right) \quad \text { a.e. in }[0, T], \\
\alpha(0) & =\alpha(T), \quad \alpha^{\prime}(0) \geq \alpha^{\prime}(T) ; \\
\beta^{\prime \prime}(t) & \leq f\left(t, \beta(t), \quad \beta^{\prime}(t)\right) \quad \text { a.e. in }[0, T], \\
\beta(0) & =\beta(T), \quad \beta^{\prime}(0) \leq \beta^{\prime}(T) .
\end{aligned}
$$

Then the periodic boundary value problem

$$
\begin{gathered}
x^{\prime \prime}(t)=f\left(t, x(t), x^{\prime}(t)\right), \\
x(0)=x(T), \quad x^{\prime}(0)=x^{\prime}(T),
\end{gathered}
$$

has at least one solution $x(t)$ such that $\alpha(t) \leq x(t) \leq \beta(t)$, for all $t \in[0, T]$.

Remark. According to (a) and (b) we say that $\alpha(t)$ is a lower solution, and $\beta(t)$ is an upper solution, of problem (2). This proposition is a particular case of results obtained by Adje [1]. We give a complete proof for convenience of the reader. 
Proof. We adapt the proof given by Mawhin [5, Theorem 1.1]. Using (iv) and (1) we fix a function $m \in L^{1}(0, T)$ such that for any $y$ with $|y| \leq 1$ we have

$$
\max \{|f(t, \alpha(t), y)|,|f(t, \beta(t), y)|, h(t), L(t)\}<m(t) .
$$

Consider the modified problem

$$
\begin{gathered}
x^{\prime \prime}=F\left(t, x, x^{\prime}\right), \\
x(0)=x(T), \quad x^{\prime}(0)=x^{\prime}(T),
\end{gathered}
$$

where $F:[0, T] \times \mathbf{R} \times \mathbf{R} \rightarrow \mathbf{R}$ is defined by

$$
\begin{aligned}
F(t, x, y) & =f(t, \alpha(t), y)+m(t)(x-\alpha(t)) \quad \text { if } x<\alpha(t), \\
& =f(t, x, y) \quad \text { if } \alpha(t) \leq x \leq \beta(t), \\
& =f(t, \beta(t), y)+m(t)(x-\beta(t)) \quad \text { if } x>\beta(t) .
\end{aligned}
$$

First we show that any solution of (4) satisfies $\alpha(t) \leq x(t) \leq \beta(t)$ for all $t \in[0, T]$ so that it is in fact a solution of (2). To see this we argue by contradiction. Suppose that $\max (x-\beta)=x\left(t_{0}\right)-\beta\left(t_{0}\right)>0$. If $t_{0} \in(0, T)$ this implies that the functions $x(t)-\beta(t)$ and

$$
-\left|x^{\prime}(t)-\beta^{\prime}(t)\right|+x(t)-\beta(t)
$$

are strictly positive in some interval $\left(t_{0}-\varepsilon, t_{0}+\varepsilon\right)$. Thus we obtain a.e. in this interval

$$
\begin{aligned}
x^{\prime \prime}(t) & =f\left(t, \beta(t), x^{\prime}(t)\right)+m(t)(x(t)-\beta(t)) \\
& \geq f\left(t, \beta(t), \beta^{\prime}(t)\right)-m(t)\left|x^{\prime}(t)-\beta^{\prime}(t)\right|+m(t)(x(t)-\beta(t)) \\
& \geq f\left(t, \beta(t), \beta^{\prime}(t)\right) \geq \beta^{\prime \prime}(t),
\end{aligned}
$$

where we have used (1) and the fact that $L(t) \leq m(t)$. This contradicts the fact that $x-\beta$ has a local maximum at $t_{0}$. If $t_{0}=0$, then since $x$ is $T$-periodic the maximum is attained at $T$ as well, and we obtain

$$
x^{\prime}(0)-\beta^{\prime}(0) \leq 0 \leq x^{\prime}(T)-\beta^{\prime}(T)
$$

which, according to (b), implies $\beta^{\prime}(0)=\beta^{\prime}(T)$. Considering the $C^{1}$-extensions of $x(t)$ and $\beta(t)$ we reach a contradiction as above. Hence $x(t) \leq \beta(t)$ for all $t \in[0, T]$. Clearly, a similar argument applies to prove that $\alpha(t) \leq x(t)$ for all $t \in[0, T]$.

It remains to prove that (4) has at least one solution. To this end, consider the homotopy

$$
\begin{gathered}
x^{\prime \prime}=(1-\lambda) m(t) x+\lambda F\left(t, x, x^{\prime}\right), \\
x(0)=x(T), \quad x^{\prime}(0)=x^{\prime}(T),
\end{gathered}
$$

where $0 \leq \lambda \leq 1$. By well-known results, it suffices as in [5] to obtain a priori estimates (in $C^{1}$-norm) for the possible solutions $x(t)$ of (5). In fact using (1) and $(3)$ we can write

$$
\begin{aligned}
\left|x^{\prime \prime}(t)\right| & =(1-\lambda) m(t)|x(t)|+\lambda\left[m(t)\left(1+\left|x^{\prime}(t)\right|\right)+m(t)(|x(t)|+M)\right] \\
& \leq m(t)\left(M+1+|x(t)|+\left|x^{\prime}(t)\right|\right),
\end{aligned}
$$


where $M=\max \left\{\|\alpha\|_{\infty},\|\beta\|_{\infty}\right\}$. The estimate on $\left\|x^{\prime}\right\|_{\infty}$ will follow from Gronwall's inequality provided we can bound $\|x\|_{\infty}$. Let us prove that

$$
\|x\|_{\infty} \leq M+1 .
$$

Suppose that this is not the case. Then there exists $t_{0} \in[0, T]$ such that

$$
\|x\|_{\infty}=\left|x\left(t_{0}\right)\right|>M+1 .
$$

If $x\left(t_{0}\right)>0$, we can fix an interval $\left(t_{0}-\varepsilon, t_{0}+\varepsilon\right)$ where $\left|x^{\prime}(t)\right|<1$ and $x(t)>\beta(t)+1$ so that a.e. in this interval we have

$$
x^{\prime \prime}(t)=(1-\lambda) m(t) x(t)+\lambda\left[f\left(t, \beta(t), x^{\prime}(t)\right)+m(t)(x(t)-\beta(t))\right],
$$

where according to (3) the bracket is larger than $-m(t)+m(t)=0$. This shows that, for any $\lambda \in[0,1]$, we have $x^{\prime \prime}(t)>0$ a.e. in $\left(t_{0}-\varepsilon, t_{0}+\varepsilon\right)$. Hence $x\left(t_{0}\right)$ cannot be a maximum. A similar argument applies if $x\left(t_{0}\right)<0$. This contradiction proves $(6)$. Therefore the proof is complete.

Remark. If the function $f$ is continuous, the estimate on $x^{\prime}(t)$ may be obtained via a Nagumo condition (cf. [5]). Here we use instead assumption (1) which, in some sense, is less general, but is adequate to our purpose. More general conditions of this type may be found in [1].

\section{Attractive ForCes}

"Solutions" of $(0)$ are always understood as (strictly) positive functions such that $(0)$ is satisfied in the usual $W^{2,1}(0, T)$-sense.

Let us introduce the following assumptions:

$\left(\mathrm{G}_{1}\right) \lim _{u \rightarrow 0^{+}} g(t, u)=+\infty$ uniformly in $t \in[0, T]$,

$\left(\mathrm{G}_{2}\right)$ there exist $R>0$ and $g_{0} \in L^{1}(0, T)$ such that $g(t, u) \leq g_{0}(t)$ if $u \geq R$ and $t \in[0, T]$.

Theorem 1. Assume (A), $\left(\mathrm{G}_{1}\right),\left(\mathrm{G}_{2}\right)$ hold and in addition

$$
\int_{0}^{T} g_{0}(t) d t \leq \int_{0}^{T} h(t) d t
$$

and there exists $M \in \mathbf{R}$ such that, for all $t \in[0, T]$,

$$
h(t) \leq M .
$$

Then the problem (0) has at least one solution.

By a ( $T$-periodic) generalized solution of $(0)$ we mean a continuous function $u(t)$ such that: (i) $u(0)=u(T)$, (ii) $u(t) \geq 0$ for all $t \in[0, T]$, (iii) the set $u^{-1}(0)$ has zero measure; (iv) given any component $J$ of $[0, T] \backslash u^{-1}(0)$, we have $u \in W^{2,1}(J)$ and $u(t)$ satisfies equation (0) in the $W^{2,1}(J)$-sense. The use of this broader concept of "solution" is useful if we wish to discard the assumption (8). 
Theorem 2. Assume (A), $\left(\mathrm{G}_{1}\right),\left(\mathrm{G}_{2}\right)$ and

$$
\int_{0}^{T} g_{0}(t) d t<\int_{0}^{T} h(t) d t
$$

In addition, let there exist $\varepsilon>0$ and $B \in L^{1}(0, T)$ such that, for all $t \in[0, T]$ and $u \in(0, \varepsilon)$, we have

$$
u g(t, u) \leq B(t)
$$

Then problem (0) has a generalized solution.

Proof of Theorem 1. By condition $\left(\mathrm{G}_{1}\right)$ and (8) we can choose a constant $\alpha>0$ sufficiently small so that for all $t \in[0, T]$

$$
g(t, \alpha) \geq M,
$$

where $M$ is the upper bound for $h(t)$ in (8). Clearly, $\alpha(t) \equiv \alpha$ is a lower solution of (0). To construct an upper solution, set, for each $b \in L^{1}(0, T)$,

$$
\bar{b}=\frac{1}{T} \int_{0}^{T} b(t) d t .
$$

Fix a constant $C=T\|\varphi\|_{1}+R+\alpha$, where $\varphi(t)=\left(g_{0}(t)-h(t)\right)-\left(\overline{g_{0}}-\bar{h}\right)$. We claim that there exists a solution $\beta_{0} \in W^{2,1}(0, T)$ of the following problem:

$$
\begin{gathered}
\beta_{0}^{\prime \prime}+f\left(\beta_{0}+C\right) \beta_{0}^{\prime}+\varphi(t)=0, \\
\beta_{0}(0)=\beta_{0}(T), \quad \beta_{0}^{\prime}(0)=\beta_{0}^{\prime}(T), \\
\bar{\beta}_{0}=0 .
\end{gathered}
$$

According to well-known results of degree theory, this follows from considering the homotopy

$$
\begin{gathered}
\beta^{\prime \prime}+\lambda f(\beta+C) \beta^{\prime}+\varphi(t)=0, \\
\beta(0)=\beta(T), \quad \beta^{\prime}(0)=\beta^{\prime}(T), \\
\bar{\beta}=0
\end{gathered}
$$

$(0 \leq \lambda \leq 1)$, if we can show that solutions of (12) are bounded, say, in $H^{1}(0, T)$. Now multiplying (12) by $\beta$ and integrating we get

$$
\left\|\beta^{\prime}\right\|_{2}^{2}=\int_{0}^{T} \varphi \beta \leq\|\varphi\|_{1}\|\beta\|_{\infty} \leq \sqrt{T}\|\varphi\|_{1}\left\|\beta^{\prime}\right\|_{2},
$$

so that $\left\|\beta^{\prime}\right\|_{2} \leq \sqrt{T}\|\varphi\|_{1}$ and finally $\|\beta\|_{\infty} \leq T\|\varphi\|_{1}$. Thus the claim is proved and moreover we know that (11) has a solution $\beta_{0}$ such that $\left\|\beta_{0}\right\|_{\infty} \leq T\|\varphi\|_{1}$. Setting $\beta=\beta_{0}+C$, it follows that

$$
\beta^{\prime \prime}+f(\beta) \beta^{\prime}+\varphi=0
$$

and $\beta$ is a $T$-periodic function such that $\beta(t) \geq R+\alpha$. In particular, using $\left(G_{2}\right)$ and $(7)$ we obtain

$$
g(t, \beta(t))-h(t) \leq g_{0}(t)-h(t)=\varphi(t)+\overline{g_{0}}-\bar{h} \leq \varphi(t)
$$


so that, by virtue of (13), $\beta$ is the desired upper solution. Clearly, equation (0) and the functions $\alpha$ and $\beta$ satisfy all the requirements in the hypothesis of Proposition 1, so that the proof is complete.

Remark. Condition $\left(\mathrm{G}_{1}\right)$ can be weakened in such a way that we are still able to construct a lower solution of $(0)$. In fact replace $\left(G_{1}\right)$ by the following assumption:

$\left(\mathrm{G}_{1}\right)^{\prime}$. There exists $r>0$ such that for all $u \in(0, r)$ we have $g(t, u) \geq g_{0}(t)$, and either

$$
\overline{g_{0}} \geq h(t) \quad(t \in[0, T]) \text { and }\left\|g_{0}-\overline{g_{0}}\right\|_{1}<r / 2 T,
$$

or

$$
\overline{g_{0}} \geq \bar{h} \text { and }\left\|g_{0}-h-\left(\overline{g_{0}}-\bar{h}\right)\right\|_{1}<r / 2 T .
$$

It is easy to show (by an argument similar to one used in the above proof) that there exists a solution $\alpha(t)$ of

$$
\begin{gathered}
\alpha^{\prime \prime}+f(\alpha) \alpha^{\prime}+g_{0}-\overline{g_{0}}=0, \\
\bar{\alpha}=\frac{r}{2}, \quad \alpha(0)=\alpha(T), \quad \alpha^{\prime}(0)=\alpha^{\prime}(T),
\end{gathered}
$$

satisfying in addition $\left\|\alpha^{\prime}\right\|_{2} \leq T^{1 / 2}\left\|g_{0}-\overline{g_{0}}\right\|_{1}$ and $\|\alpha-r / 2\|_{\infty} \leq T\left\|g_{0}-\overline{g_{0}}\right\|_{1}$. It follows from $\left(G_{1}\right)^{\prime}$ that $\alpha$ takes values in $(0, r)$ and hence it is a lower solution of $(0)$.

Proof of Theorem 2. For each $n \in \mathbf{N}$, let $h_{n}=\inf (h, n)$. From Theorem 1 it follows that the truncated problem

$$
\begin{gathered}
u_{n}^{\prime \prime}+f\left(u_{n}\right) u_{n}^{\prime}+g\left(t, u_{n}\right)=h_{n}(t), \\
u_{n}(0)=u_{n}(T), \quad u_{n}^{\prime}(0)=u_{n}^{\prime}(T),
\end{gathered}
$$

has a solution $u_{n} \in W^{2,1}(0, T)$ which is strictly positive. To obtain estimates on $u_{n}$ we first remark that, for $n$ sufficiently large, we cannot have $u_{n}(t) \geq R$ for all $t \in[0, T]$, since

$$
\int_{0}^{T} g_{0}(t) \geq \int_{0}^{T} g\left(t, u_{n}(t)\right) d t=\int_{0}^{T} h_{n}(t) d t
$$

and this would contradict (9). Hence there exists $t_{n}$ such that $u_{n}\left(t_{n}\right) \leq R$. On the other hand

$$
\left\|u_{n}^{\prime}\right\|_{2}^{2}=\int_{0}^{T}\left[g\left(t, u_{n}\right) u_{n}-h(t) u_{n}\right] d t
$$

which, on the basis of $(A),\left(G_{2}\right)$ and (10) implies

$$
\left\|u_{n}^{\prime}\right\|_{2}^{2} \leq c_{1}\left\|u_{n}\right\|_{\infty}+c_{2} \leq c_{1}\left(R+\sqrt{T}\left\|u_{n}^{\prime}\right\|_{2}\right)+c_{2}
$$

where $c_{1}, c_{2}$ are positive constants independent of $n$. Thus for some subsequence, still labelled $\left(u_{n}\right)$, we have

$$
u_{n} \rightarrow u \quad \text { weakly in } H^{1}(0, T) \text { and uniformly in }[0, T] .
$$


Of course, $u(t) \geq 0$ for all $t \in[0, T]$. Set

$$
\begin{aligned}
\tilde{g}(t) & =g(t, u(t)) \quad \text { if } u(t)>0, \\
& =+\infty \quad \text { if } u(t)=0 .
\end{aligned}
$$

Our hypothesis (A) on $g$ and $\left(G_{1}\right)$ show that the sequence of functions $g\left(t, u_{n}(t)\right)$ is bounded below by some integrable function. Then using Fatou's lemma we have

$$
\int_{0}^{T} \tilde{g}(t) d t \leq \lim \int_{0}^{T} g\left(t, u_{n}(t)\right) d t=\lim \int_{0}^{T} h_{n}(t) d t=\int_{0}^{T} h(t) d t,
$$

showing that $\tilde{g}(t)$ is finite almost everywhere in $(0, T)$. This proves that the set $u^{-1}(0)$ has zero measure. Now let $J$ be any component of $[0, T] \backslash u^{-1}(0)$. Taking an arbitrary test function $\varphi \in C_{0}^{\infty}(J)$, we obtain from (14)

$$
\int_{0}^{T}\left[-u_{n}^{\prime} \varphi^{\prime}+f\left(u_{n}\right) u_{n}^{\prime} \varphi+g\left(t, u_{n}\right) \varphi\right] d t=\int_{0}^{T} h_{n} \varphi
$$

and, since $g\left(t, u_{n}(t)\right) \rightarrow g(t, u(t))$ in $L^{1}(\operatorname{supp} \varphi)$,

$$
\int_{0}^{T}\left[-u^{\prime} \varphi^{\prime}+f(u) u^{\prime} \varphi+g(t, u(t)) \varphi\right] d t=\int_{0}^{T} h \varphi .
$$

But we know that $g(t, u(t))=\tilde{g}(t)$ is $L^{1}(0, T)$ so that $u \in W^{2,1}(J)$ and the result follows.

\section{REPELLING FORCES}

In this section we consider the problem

$$
\begin{gathered}
u^{\prime \prime}+f(u) u^{\prime}+g(u)=h(t), \\
u(0)=u(T), \quad u^{\prime}(0)=u^{\prime}(T),
\end{gathered}
$$

where $f \in C(\mathbf{R}, \mathbf{R})$ and $g \in C((0,+\infty), \mathbf{R})$. We introduce the following assumptions:

$\left(\mathrm{F}_{1}\right)$ There exists $a>0$ such that, for every $u>0$,

$$
f(u) \geq a \quad \text { [respectively } f(u) \leq-a \text { ] }
$$

$\left(\mathrm{G}_{3}\right)$ There exists $R>1$ such that, for every $u>R$,

$$
g(u)>\bar{h} \text {. }
$$

$\left(\mathrm{G}_{4}\right)$ There exists $r<1$ such that, for every $u \in(0, r)$,

$$
g(u)<\bar{h} .
$$

$$
\int_{0}^{1} g(u) d u=-\infty \text {. }
$$


Theorem 3. Assume $h \in L^{2}(0, T)$ and let $f$ and $g$ be real continuous functions in $\mathbf{R}$ and in $(0,+\infty)$, respectively, satisfying $\left(\mathrm{F}_{1}\right),\left(\mathrm{G}_{3}\right),\left(\mathrm{G}_{4}\right)$ and $\left(\mathrm{G}_{5}\right)$. Then the problem (15) has at least one solution.

Proof. Consider the homotopy

$$
\begin{gathered}
u^{\prime \prime}+f(u) u^{\prime}+g_{\lambda}(u)=h_{\lambda}(u), \\
u(0)=u(T), \quad u^{\prime}(0)=u^{\prime}(T),
\end{gathered}
$$

where $g_{\lambda}(u)=(1-\lambda)\left(\bar{h}+1-u^{-1}\right)+\lambda g(u), h_{\lambda}(t)=(1-\lambda) \bar{h}+\lambda h(t)$. Note that $g_{\lambda}$ satisfies $\left(\mathrm{G}_{3}\right),\left(\mathrm{G}_{4}\right)$ and $\left(\mathrm{G}_{5}\right)$ uniformly in $\lambda \in[0,1]$ and that $\bar{h}_{\lambda}=\bar{h}$.

First we obtain an $L^{2}(0, T)$-bound for the derivative of any solution $u(t)$ of (16). Indeed, multiplication by $u^{\prime}$ and integration yields

$$
a\|\dot{u}\|_{2}^{2} \leq \pm \int_{0}^{T} f(u) u^{\prime 2}= \pm \int_{0}^{T} h_{\lambda} u^{\prime} \leq\|h\|_{2}\left\|u^{\prime}\right\|_{2} .
$$

Next, it follows easily from $\left(\mathrm{G}_{3}\right)$ that there exists a $t_{0}$ such that

$$
u\left(t_{0}\right)<R
$$

this, together with what has just been shown gives an upper bound for all solutions $u(t)$ of (16):

$$
u(t) \leq u\left(t_{0}\right)+\int_{t_{0}}^{t}\left|u^{\prime}\right|<R+T^{1 / 2}\|h\|_{2} a^{-1} \equiv M .
$$

Now we are going to obtain a uniform bound on $u^{\prime}(t)$. To this purpose, extend $u$ as a $T$-periodic function and let $u\left(t_{1}\right)$ be any extremum of $u$. For $t \geq t_{1}$ it turns out that

$$
u^{\prime}(t)=\int_{t_{1}}^{t} u^{\prime \prime}=-\int_{t_{1}}^{t} f(u) u^{\prime}-\int_{t_{1}}^{t} g_{\lambda}(u)+\int_{t_{1}}^{t} h_{\lambda} .
$$

Since $\|u\|_{\infty}<M,\left(\mathrm{G}_{4}\right)$ implies that $g_{\lambda}(u)$ is bounded above. Hence the righthand side is bounded from below, say

$$
u^{\prime}(t)>-M_{1} \text {. }
$$

Similarly, for $t \leq t_{1}$,

$$
u^{\prime}(t)=\int_{t}^{t_{1}} f(u) u^{\prime}+\int_{t}^{t_{1}} g_{\lambda}(u)+\int_{t}^{t_{1}} h_{\lambda} \leq M_{1} .
$$

This shows that

$$
\left\|u^{\prime}\right\|_{\infty}<M_{1} .
$$

On the basis of $\left(\mathrm{G}_{4}\right)$ we can choose $t_{2}$ such that $u\left(t_{2}\right)>r$. Multiplying (16) by $u^{\prime}$ and integrating in an interval $\left[t_{2}, t\right]$ we get

$$
\frac{u^{\prime}(t)^{2}}{2}-\frac{u^{\prime}\left(t_{2}\right)^{2}}{2}+\int_{t_{2}}^{t} f(u) u^{\prime 2}+\int_{t_{2}}^{t} g_{\lambda}(u) u^{\prime}=\int_{t_{2}}^{t} h_{\lambda} u^{\prime}
$$


so that, using the bounds on $u(t),\left\|u^{\prime}\right\|_{\infty}$ and (17), we see that

$$
\int_{t_{2}}^{t} g_{\lambda}(u) u^{\prime}=\lambda \int_{u\left(t_{2}\right)}^{u(t)} g(u) d u+(1-\lambda)\left[\ln \frac{u\left(t_{2}\right)}{u(t)}+(\bar{h}+1)\left(u(t)-u\left(t_{2}\right)\right)\right]
$$

is bounded. Assumption $\left(\mathrm{G}_{5}\right)$ then shows that there exists $\varepsilon>0$ such that, for any such solution $u(t)$,

$$
u(t)>\varepsilon, \quad t \in[0, T] .
$$

The estimates thus obtained enable us to apply coincidence degree to the homotopy (16). Define the operators

$$
\begin{aligned}
& L: D(L) \subset H^{2}(0, T) \rightarrow L^{2}(0, T), \\
& N:[0,1] \times \bar{\Omega} \rightarrow L^{2}(0, T),
\end{aligned}
$$

where

$$
\begin{gathered}
D(L)=\left\{u \in H^{2}(0, T): u(0)=u(T), u^{\prime}(0)=u^{\prime}(T)\right\}, \\
L u=u^{\prime \prime} \quad \text { if } u \in D(L), \\
\Omega=\left\{u \in C^{1}([0, T]): \varepsilon<u(t)<M,\left\|u^{\prime}\right\|_{\infty}<M_{1}\right\}, \\
N(\lambda, u)=h_{\lambda}-f(u) u^{\prime}-g_{\lambda}(u) .
\end{gathered}
$$

From well-known properties of the coincidence degree [5] one has

$$
d_{L}(L-N(\lambda, \cdot), \Omega)=d_{L}(L-N(0, \cdot), \Omega)= \pm d_{B}\left(\bar{h}-g_{0}(u), D\right)= \pm 1 \text {. }
$$

Here $d_{B}$ denotes the Brower degree, $\left.D=\right] \varepsilon, M[$, and we have used the fact that, by virtue of $\left(F_{1}\right)$, the only solution of $(16)$ for $\lambda=0$ is the constant solution $u=1$. This shows that (16) is solvable for $\lambda=1$ and so the proof is complete.

It is easy to see that all the estimates in the above proof remains valid if we replace $\left(\mathrm{F}_{1}\right)$ by

$\left(\mathrm{G}_{6}\right)$ There exists a constant $C>0$ such that, for every $t \in[0, T]$ and every $u>0$,

$$
g(u)-h(t) \leq C .
$$

The only difference is that the bound on $\left\|u^{\prime}\right\|_{2}$ now follows from the inequalities

$$
\int_{0}^{T} u^{\prime 2} \leq \int_{0}^{T}(g(u)-h) u \leq k\|u\|_{1}
$$

and

$$
u(t) \leq R+T^{1 / 2}\left\|u^{\prime}\right\|_{2}
$$

(obtained as in the above proof, using $\left(\mathrm{G}_{3}\right)$ ). Hence we can state:

Theorem 4. Assume $h \in L^{2}(0, T)$ and let $f, g$ be real continuous functions in $\mathbf{R}$ and in $(0,+\infty)$, respectively, satisfying $\left(\mathrm{G}_{3}\right),\left(\mathrm{G}_{4}\right),\left(\mathrm{G}_{5}\right)$ and $\left(\mathrm{G}_{6}\right)$. Then the problem (15) has at least one solution. 


\section{REFERENCES}

1. A. Adje, Sur et sous solutions dans les équations différentielles discontinues avec conditions aux limites non lineaires, These de doctorat, Louvain-la-Neuve, 1987.

2. A. Bahri and P. Rabinowitz, A minimax method for a class of Hamiltonian systems with singular potentials, CMS Technical Summary Report, 88-8, 1987.

3. P. Habets and L. Sanchez, Periodic solutions of dissipative dynamical systems with singular potentials, Differential and Integral Equations (to appear).

4. A. C. Lazer and S. Solimini, On periodic solutions of nonlinear differential equations with singularities, Proc. Amer. Math. Soc. 99 (1987), 109-114.

5. J. Mawhin, Points fixes, points critiques et problemes aux limites, Semin. Math. Sup. no. 92, Presses Univ. de Montréal, 1985.

Institut de Mathématique Pure et Appliquée, Chemin du Cycloton, 2, B-1348 LouvainLA-Neuve, BeLgium

inic/CMaf, Avenida Professor Gama Pinto, 2, P-1699 Lisboa Codex, Portugal 